Research Article

\title{
Correlation between Colon Polyps and Metabolic Syndrome and HP Infection Status
}

\author{
Lijuan Huang $\mathbb{D}$, Lihong Wu, Qiaohua Qiao, and Lizheng Fang $\mathbb{D}$ \\ Department of General Practice, Sir Run Run Shaw Hospital, Zhejiang University School of Medicine, Hangzhou, \\ Zhejiang Province, China \\ Correspondence should be addressed to Lijuan Huang; huanglijuan_lgc@163.com
}

Received 27 March 2019; Revised 19 May 2019; Accepted 23 May 2019; Published 4 June 2019

Academic Editor: Chiara Ricci

Copyright (C) 2019 Lijuan Huang et al. This is an open access article distributed under the Creative Commons Attribution License, which permits unrestricted use, distribution, and reproduction in any medium, provided the original work is properly cited.

Background. This study investigated the relationships among the characteristics of colon polyps and potential risk factors, including metabolic condition, CEA level, uric acid level, and Helicobacter pylori (Hp) infection status. Method. Clinical data from patients who received colonoscopy were collected and analyzed, including patients' gender, age, polyp pathology, metabolic syndrome (MS) status, CEA level, uric acid level, and Hp infection status. Patients were divided into a polyp group and a control group based on whether they presented with colon polyps. Then, clinical data were compared between the two groups to identify any differences between the groups and their relationships to colon polyps. Result. Compared with the control group, the polyp group had significant differences in patient gender, body mass index (BMI), waistline, blood pressure, fasting blood glucose level, blood lipid level, and uric acid level $(p<0.05)$, but there were no significant differences in LDL and CEA levels $(p>0.05)$. Patients with MS or a uric acid level $>340 \mathrm{mg} / \mathrm{dl}$ had a greater tendency to develop colon polyps but this was not statistically significant. Conclusion. The incidence of colon polyps may be associated with MS and uric acid levels, but further studies are warranted to confirm this conclusion.

\section{Introduction}

Colon polyps, especially adenomatous polyps, have been widely regarded as precancerous lesions that are caused by various pathogenic factors, including heredity, inappropriate diet habits, and infections [1]. Among these pathogenic factors, Helicobacter pylori (Hp) infection plays an important role. HP infection has been reported to be associated with the pathogenesis of colon polyps [2]. Data have shown that the incidence of colon polyps has dramatically increased in the past 20 years in the Asia-Pacific area, which may have been associated with a high-fat, high-protein diet and a lack of physical exercise $[3,4]$. There have also been multiple studies showing the relationship between colon polyps and metabolic syndrome- (MS-) associated indexes, including blood lipid level, glucose level, and BMI [3, 5-7]. In recent years, the detection rate of colon polyps has grown due to the increasing use of colonoscopy, and it has been reported that more than $85 \%$ of all colon cancer cases transform from colon polyps [8]. However, the association between the pathology and site of colon polyps and HP infection is poorly investigated. Besides, considering the metabolic factors may influence the formation of colon polyps, we discussed whether uric acid levels and MS status could serve as risk factors for the occurrence of colon polyp. CEA level was reported as a marker for the occurrence of colon polyps and was examined in our study [9].

\section{Methods}

Patients were identified from Sir Run Run Shaw Hospital, Zhejiang University, from Jan 2010 to May 2014. The included patients should have undergone gastroscopy, colonoscopy, C13 breath tests, and routine blood tests. Patients presenting colon polyps were classified as the polyp group, and those who did not present with colon polyps were classified as the control group. A C13 breath test was employed to test for HP infection, and those with positive results were considered $\mathrm{Hp}$ positive. Nearly all detected polyps were removed and colon polyp samples were 
TABLE 1: Baseline characteristics of patients in the polyp group and the control group.

\begin{tabular}{lccc}
\hline Variables & Controls $(N=334)$ & Cases $(N=159)$ & $p$ \\
\hline Gender (male) & $239(71.56)$ & $138(86.79)$ & 0.0002 \\
Family history of cancer (yes) & $77(23.05)$ & $41(25.79)$ & 0.5063 \\
Age & $46.22 \pm 8.13$ & $50.10 \pm 8.29$ & $<0.0001$ \\
BMI & $24.29 \pm 3.06$ & $25.17 \pm 3.17$ & 0.0032 \\
Waist $^{\text {a }}$ & $85.41 \pm 9.33$ & $88.94 \pm 10.02$ & 0.0002 \\
SBP & $116.17 \pm 12.33$ & $122.85 \pm 13.76$ & $<0.0001$ \\
DBP & $70.24 \pm 9.11$ & $75.25 \pm 10.48$ & $<0.0001$ \\
FPG & $4.85(4.50,5.20)$ & $5.07(4.72,5.44)$ & $<0.0001$ \\
TG & $1.39(0.91,2.11)$ & $1.72(1.17,2.42)$ & 0.0013 \\
TC & $4.69 \pm 0.93$ & $4.87 \pm 1.01$ & 0.0441 \\
HDL & $1.15 \pm 0.28$ & $1.07 \pm 0.24$ & 0.0032 \\
LDL & $2.64 \pm 0.83$ & $2.80 \pm 0.88$ & 0.0535 \\
CEA & $1.43(0.82,2.13)$ & $1.64(0.82,2.52)$ & 0.0609 \\
UA & $342.40 \pm 95.78$ & $366.03 \pm 80.23$ & 0.0044 \\
\hline
\end{tabular}

${ }^{*}$ Categorial variable, $n(\%)$; continuous variable, mean \pm SD/median (p25, p75). ${ }^{\mathrm{a}}$ Data for two cases and six controls were unknown. ${ }^{\mathrm{b}}$ Data for 12 cases were unknown.

processed, histological slices were made, and HE staining was used for pathologic diagnosis. Pathological classification of colon polyps included inflammatory polyp (I), proliferative polyp (P), adenoma (A), and cancer (C); sites of colon polyps included the left colon (L) (including the rectum and sigmoid and descending colon), transverse colon ( $\mathrm{T}$ ) (including splenic flexure, transverse colon, and hepatic flexure), and right colon $(\mathrm{R})$ (including the ascending colon and ileocecal junction). Polyp size was assessed according to the Yamada standard and classified as Yamada type I, II, III, or IV; the number of polyps was classified as single or multiple. Our study was performed after obtaining informed consent from the patients and approval from the Ethics Committee of Sir Run Run Shaw Hospital, Zhejiang University.

Patients with chronic gastritis, digestive ulcers, and gastric cancer and who underwent previous gastric surgery were excluded by gastroscopy. Patients who underwent Hp eradication therapy or those with ulcerative colitis, Crohn's disease, or familial adenomatous polyps were also excluded.

SPSS 19.0 was employed for statistical analysis, and quantitative data were expressed in the form of $(X \pm S)$. A $t$-test was used to compare the differences. Enumeration data were expressed as $n(\%)$. The chi-square test was used to test the difference and $p<0.05$ was accepted as statistically significant. Nonconditional logistic regression was expressed using the odds ratio and a 95\% confidence interval.

\section{Results}

3.1. Baseline Characteristics of Patients from the Polyp Group and the Control Group. In this study, 159 patients were enrolled in the polyp group and 334 patients were enrolled in the control group. The average age of the patients was $50.10 \pm 8.29$ years for the polyp group and $46.22 \pm 8.13$ years for the control group; the difference was significant $(p<$ $0.0001)$. Significant differences were also found in gender,
BMI, waistline, blood pressure, fasting blood glucose level, blood lipid level, and uric acid level $(p<0.05)$, but no significant difference in LDL and CEA was detected between the two groups $(p>0.05)$ (Table 1$)$.

3.2. Relationship between Hp Infection/MS and Colon Polyps. For patients with multiple colon polyps, the largest polyp was assessed for location and Yamada classification, and the most advanced pathological type of all polyps was taken as the pathological status of the patient. Comparing the $\mathrm{H}$. pylorigroup and the $\mathrm{H}$. pylori+ group, there were no significant differences in polyp number, site, and pathology $(p>0.05)$. In the subgroup analysis, polyp patients with MS had significantly more polyps than those without MS, and no significant difference was detected in polyp size, location, and pathological type in the subgroups described previously (Tables 2(a) and $2(\mathrm{~b}))$.

3.3. MS Status, CEA Level, Uric Acid Level, and Hp Infection Status in Patients with Colon Polyps. Within the 157 colon polyp patients, 63 had metabolic syndrome (MS) (40.13\%), whereas 73 of the $334(22.26 \%)$ patients in the control group had MS; the difference was significant $(p=0.0023)$. For all enrolled patients, subgroup analysis showed that patients with MS had a significantly greater chance of developing colon polyps $(\mathrm{OR}=2.16,95 \% \mathrm{CI}=1.32-3.54)$.

In this study, we also found that the polyp group and the control group had no significant difference in CEA level, uric acid level, and $\mathrm{Hp}$ infection status. If we divide all enrolled patients by the median uric acid level $(340 \mathrm{mg} / \mathrm{dl})$ into a high uric acid level group ( $\geq 340 \mathrm{mg} / \mathrm{dl})$ and a low uric acid level group $(<340 \mathrm{mg} / \mathrm{dl})$, the relative risk of developing colon polyps in the former group was 0.83 times higher than that in the latter group $(95 \% \mathrm{CI}=0.52-1.3)$. Similarly, for all patients, $\mathrm{Hp}$-positive patients had a relative risk 
TABLE 2

(a) Polyp parameters and $\mathrm{Hp}$ infection status

\begin{tabular}{lccc}
\hline & H. pylori+ & H. pylori- & $p$ \\
\hline No. of polyps & & & 0.9085 \\
$\quad$ None & $208(62.28)$ & $126(37.72)$ & \\
$\quad$ Single & $66(60.55)$ & $43(39.45)$ & \\
$\quad$ Multiple & $32(64.00)$ & $18(36.00)$ & \\
Polyp size & & & 0.3518 \\
I & $67(65.05)$ & $36(34.95)$ & \\
II & $12(48.00)$ & $13(52.00)$ & \\
III & $14(66.67)$ & $7(33.33)$ & \\
IV & $5(50.00)$ & $5(50.00)$ & \\
Polyp location & & & 0.5707 \\
L & $74(63.79)$ & $42(36.21)$ & \\
R & $12(52.17)$ & $11(47.83)$ & \\
T & $12(60.00)$ & $8(40.00)$ & \\
Polyp pathology & & & 0.6865 \\
A & $57(58.76)$ & $40(41.24)$ & \\
P & $17(60.71)$ & $11(39.29)$ & \\
I & $23(69.70)$ & $10(30.30)$ & \\
C & $1(100.00)$ & $0(0.00)$ & \\
\hline
\end{tabular}

${ }^{* a}$ Fisher's exact test.

(b) Polyp parameters and MS status

\begin{tabular}{|c|c|c|c|}
\hline \multirow{2}{*}{ Polyp } & \multicolumn{2}{|c|}{ MS } & \multirow[b]{2}{*}{$p$} \\
\hline & No & Yes & \\
\hline No. of polyps & & & 0.0002 \\
\hline None & $255(77.74)$ & $73(22.26)$ & \\
\hline Single & $66(61.11)$ & $42(38.89)$ & \\
\hline Multiple & $28(57.14)$ & $21(42.86)$ & \\
\hline Polyp size & & & 0.2315 \\
\hline I & $63(61.76)$ & $39(38.24)$ & \\
\hline II & $14(58.33)$ & $10(42.67)$ & \\
\hline III & $14(66.67)$ & $7(33.33)$ & \\
\hline IV & $3(30.00)$ & $7(70.00)$ & \\
\hline Polyp location & & & 0.5755 \\
\hline $\mathrm{L}$ & $69(60.53)$ & $45(39.47)$ & \\
\hline $\mathrm{R}$ & $15(65.22)$ & $8(34.78)$ & \\
\hline $\mathrm{T}$ & $10(50.00)$ & $10(50.00)$ & \\
\hline Polyp pathology ${ }^{\mathrm{a}}$ & & & 0.6926 \\
\hline A & $59(61.46)$ & $37(38.54)$ & \\
\hline $\mathrm{P}$ & $17(60.71)$ & $11(39.29)$ & \\
\hline I & $18(56.25)$ & $14(43.75)$ & \\
\hline $\mathrm{C}$ & $0(0.00)$ & $1(100.00)$ & \\
\hline
\end{tabular}

${ }^{* a}$ Fisher's exact test.

of developing polyps that was 1.15 times higher than HPnegative patients $(95 \% \mathrm{CI}=0.76-1.74)$ (Table 3$)$.

3.4. Interactive Variant Analysis of All of the Related Factors. Interactive variant analysis was used to study the interrela-
TABLE 3: MS, CEA, UC, and Hp infection status in the polyp group and the control group.

\begin{tabular}{lcccc}
\hline Variables & $\begin{array}{c}\text { Controls } \\
(N=334)\end{array}$ & $\begin{array}{c}\text { Cases } \\
(N=159)\end{array}$ & OR (95\% CI $)$ & $p$ \\
\hline MS & & & & \\
No & $255(77.74)$ & $94(59.87)$ & 1.00 & - \\
Yes & $73(22.26)$ & $63(40.13)$ & $2.16(1.32,3.54)$ & 0.0023 \\
CEA & & & & \\
$<1.43$ & $167(50.00)$ & $67(42.14)$ & 1.00 & - \\
$\geq 1.43$ & $167(50.00))$ & $92(57.86)$ & $1.06(0.71,1.58)$ & 0.7867 \\
UA & & & & \\
$<340$ & $167(50.00)$ & $70(44.03)$ & 1.00 & - \\
$\geq 340$ & $167(50.00)$ & $89(55.97)$ & $0.83(0.52,1.30)$ & 0.4094 \\
H. pylori & & & & \\
$\quad$ Negative & $208(62.28)$ & $98(61.64)$ & 1.00 & - \\
Positive & $126(37.72)$ & $61(38.36)$ & $1.15(0.76,1.74)$ & 0.4990 \\
\hline
\end{tabular}

* OR adjusted for age, gender, BMI, and family history of cancer.

tionship among factors, including MS status, CEA level, uric acid level, and $\mathrm{Hp}$ infection status. The results revealed that the median value of the CEA level of the enrolled patients was 1.43 , and we defined those with $\mathrm{CEA}<1.43$ as low CEA and those with CEA $\geq 1.43$ as high CEA. Patients with MS had a higher risk of developing colon polyps independent of their CEA level, but as patients' CEA level increased, the risk of developing colon polyps also increased $(\mathrm{OR}=2.58$, $95 \% \mathrm{CI}=1.29-5.17)$; however, these two factors had no interactive effect $(\mathrm{OR}=1.22,95 \% \mathrm{CI}=0.51-2.90)$.

Compared with patients who had high uric acid level and did not have MS, patients with MS had a higher risk of developing colon polyps independent of their uric acid level; however, this risk escalated as patients' uric acid level decreased $(\mathrm{OR}=2.58,95 \% \mathrm{CI}=1.13-5.89)$. These above two factors also had no interactive effect $(\mathrm{OR}=1.35$, $95 \% \mathrm{CI}=0.53-3.44)$ (Table 4).

Overall, no significant interactive effects existed among MS, CEA level, uric acid level, and Hp infection state.

\section{Discussion}

Recently, there have been multiple studies concerning $\mathrm{Hp}$ infection and colon polyps, and the results showed that patients with adenomatous polyposis coli (APC) had a high incidence of $\mathrm{Hp}$ infection; therefore, it has been hypothesized that $\mathrm{Hp}$ infection could increase the risk of adenoma and adenocarcinoma in the colon and rectum, and their relationship was also demonstrated by the mucosal proliferation/APC/adenocarcinoma axis [10-12]. Zumkeller et al. analyzed the literature from 1991 to 2002 in a meta-analysis and showed that $\mathrm{Hp}$ infection might be associated with the pathogenesis of colon adenoma and adenocarcinoma, with an OR of 1.4 (95\% CI: 1.1-1.8) [13]. Oh et al. found that gastric cancer patients had a higher risk of colon cancer [14]. Additionally, Soylu et al. found $\mathrm{Hp}$ in samples of colon adenoma by immunohistochemistry. All of these studies suggest a potential relationship between $\mathrm{Hp}$ infection and colon cancer. 
TABLE 4: Interactive analysis among MS, CEA, UC, and Hp infection status.

\begin{tabular}{|c|c|c|c|c|c|}
\hline Variable 1 & Variable 2 & Controls $(N=334)$ & Cases $(N=159)$ & OR $(95 \%$ CI $)$ & $p$ \\
\hline$M S$ & $C E A$ & & & & \\
\hline No & $<1.43$ & $125(38.11)$ & $37(23.57)$ & 1.00 & - \\
\hline No & $\geq 1.43$ & $130(39.63)$ & $57(36.31)$ & $1.06(0.64,1.78)$ & 0.8114 \\
\hline Yes & $<1.43$ & $39(11.89)$ & $29(18.47)$ & $1.99(1.02,3.86)$ & 0.0432 \\
\hline Yes & $\geq 1.43$ & $34(10.37)$ & $34(21.66)$ & $2.58(1.29,5.17)$ & 0.0075 \\
\hline Interaction OR & & & & $1.22(0.51,2.90)$ & 0.6519 \\
\hline$M S$ & $U A$ & & & & \\
\hline No & $\geq 340$ & $114(34.76)$ & $43(27.39)$ & 1.00 & - \\
\hline No & $<340$ & $141(42.99)$ & $51(32.48)$ & $1.41(0.83,2.42)$ & 0.2067 \\
\hline Yes & $\geq 340$ & $52(15.85)$ & $46(29.30)$ & $2.46(1.38,4.40)$ & 0.0024 \\
\hline Yes & $<340$ & $21(6.40)$ & $17(10.83)$ & $2.58(1.13,5.89)$ & 0.0242 \\
\hline Interaction OR & & & & $1.35(0.53,3.44)$ & 0.5330 \\
\hline MS & H. pylori & & & & \\
\hline No & Negative & $163(49.70)$ & $61(38.85)$ & 1.00 & - \\
\hline No & Positive & $92(28.05)$ & $33(21.02)$ & $1.13(0.67,1.89)$ & 0.6518 \\
\hline Yes & Negative & $42(12.80)$ & $36(22.93)$ & $2.15(1.18,3.91)$ & 0.0124 \\
\hline Yes & Positive & $31(9.45)$ & $27(17.20)$ & $2.41(1.23,4.73)$ & 0.0106 \\
\hline Interaction OR & & & & $1.00(0.42,2.38)$ & 0.9932 \\
\hline H. pylori & $C E A$ & & & & \\
\hline Negative & $<1.43$ & $102(30.54)$ & $43(27.04)$ & 1.00 & - \\
\hline Negative & $\geq 1.43$ & $106(31.74)$ & $55(34.59)$ & $0.94(0.57,1.57)$ & 0.8230 \\
\hline Positive & $<1.43$ & $65(19.46)$ & $24(15.09)$ & $0.98(0.53,1.81)$ & 0.9438 \\
\hline Positive & $\geq 1.43$ & $61(18.26)$ & $37(23.27)$ & $1.24(0.70,2.18)$ & 0.4642 \\
\hline Interaction OR & & & & $1.34(0.59,3.05)$ & 0.4853 \\
\hline H. pylori & $U A$ & & & & \\
\hline Negative & $\geq 340$ & $102(30.54)$ & $56(35.22)$ & 1.00 & - \\
\hline Negative & $<340$ & $106(31.74)$ & $42(26.42)$ & $1.04(0.60,1.79)$ & 0.8876 \\
\hline Positive & $\geq 340$ & $65(19.46)$ & $33(20.75)$ & $0.96(0.55,1.65)$ & 0.8684 \\
\hline Positive & $<340$ & $61(18.26)$ & $28(17.61)$ & $1.55(0.81,2.93)$ & 0.1832 \\
\hline Interaction $\mathrm{OR}$ & & & & $0.64(0.28,1.47)$ & 0.2935 \\
\hline CEA & $U A$ & & & & \\
\hline$<1.43$ & $\geq 340$ & $72(21.56)$ & $36(22.64)$ & 1.00 & - \\
\hline$<1.43$ & $<340$ & $95(28.44)$ & $31(19.50)$ & $1.03(0.54,1.95)$ & 0.9338 \\
\hline$\geq 1.43$ & $\geq 340$ & $95(28.44)$ & $53(33.33)$ & $0.93(0.54,1.60)$ & 0.7969 \\
\hline$\geq 1.43$ & $<340$ & $72(21.56)$ & $39(24.53)$ & $1.30(0.70,2.42)$ & 0.4133 \\
\hline Interaction OR & & & & $0.74(0.33,1.65)$ & 0.4576 \\
\hline
\end{tabular}

${ }^{*}$ OR adjusted for age, gender, BMI, and family history of cancer. CEA (1.43) and UA (340) were determined by the corresponding median value of enrolled patients. Interaction OR: interactive effect of the multiplicative model.

There have also been studies that have presented conflicting results. For example, in a prospective study, the Hp infection rate was not significantly different between patients with colon polyps/cancer and a normal cohort, which did not support the hypothesis that the polyps were relevant to the formation of cancer [11, 15]. Additionally, Abbass et al. proposed that the incidence of colon polyp/cancer was not significantly different in $\mathrm{Hp}$-positive and $\mathrm{Hp}$-negative patients [16].

Several meta-analyses of the literature from the Western world showed that Hp infection had a certain relationship with the pathogenesis of colon polyps and that $\mathrm{Hp}$ infec- tion increased the risk of colorectal adenoma and adenocarcinoma; however, the meta-analysis drew no concrete conclusion [11, 17]. Several studies have investigated the relationship between $\mathrm{Hp}$ infection and colon polyps in the Chinese population [12]. Lin et al. launched a crosssectional study in Taiwan province and found that MS patients with $\mathrm{Hp}$ infection were more susceptible to colorectal adenoma [18].

The mechanisms of how Hp infection could induce colon polyps and carcinomas are still unclear, and research has shown that gastrin and cyclooxygenase-2 (COX-2) were involved; gastrin can regulate gastric acid secretion, promote 
the proliferation of gastric mucosa, and promote the proliferation and metastasis of gastric tumor cells $[19,20]$. COX is the rate-limiting enzyme of prostaglandin synthesis, and under pathological conditions, COX-2 promotes tumor cell proliferation, inhibits cell apoptosis, promotes the release of angiopoietin, inhibits endothelial apoptosis, and activates MMPs (matrix metalloproteinases), thus inducing tumor angiogenesis. It has been suggested that aspirin can reduce the incidence of colon adenomas and individuals who took COX-2 inhibitors had a lower incidence of colon cancer than those who did not [21]. However, there were also different conflicting data, and Selgrad et al. found that gastrin did not increase patients' risk of colorectal cancer [22]. In summary, the role of $\mathrm{Hp}$ in the pathogenesis of colon polyps is still unclear.

Besides, metabolic factors were also reported as risk factors for polyp occurrence. A cohort study involving 2244 patients indicated that visceral adipose tissue was dosedependently associated with colorectal adenoma [23]. Similarly, abdominal obesity, hypertension, and a high $\mathrm{HbAlc} \%$ increase the risk for polyps [24]. Consistent with previous reports, we found that uric acid and MS index were also strongly associated with colon polyp. Uric acid is an important damage-associated molecular pattern (PAMP) and exerts strong proinflammation effect [25]. Monosodium urate crystals stimulate inflammatory response by activation of toll-like receptor 4 , which strongly promote leukocyte to produce proinflammatory cytokines. The proinflammatory factors were known to play a critical role in regulating neoplasm formation and cancer progress [26]. Therefore, reducing serum uric acid level may decrease level of systemic inflammation and break polyp-cancer transformation.

In this study, we found that the risk of colon polyps escalated as patients' age increased and that compared to women men were more likely to have colon polyps. We also found that MS was a risk factor for colon polyps. Within MS patients, colon polyp risk escalated as CEA level increased, but the risk declined as uric acid level increased. Hp infection may increase the risk of colon polyps. This study was retrospective and had a limited sample size. Further prospective studies with a larger cohort of patients are needed to verify our hypothesis.

\section{Data Availability}

The data used to support the findings of this study are available from the corresponding author upon request.

\section{Conflicts of Interest}

The authors declare that there is no conflict of interest in the publication of this paper.

\section{References}

[1] P. J. Pickhardt, B. D. Pooler, D. H. Kim, C. Hassan, K. A. Matkowskyj, and R. B. Halberg, "The natural history of colorectal polyps: overview of predictive static and dynamic features," Gastroenterology Clinics of North America, vol. 47, no. 3, pp. 515-536, 2018.
[2] J. H. Nam, C. W. Hong, B. C. Kim et al., "Helicobacter pylori infection is an independent risk factor for colonic adenomatous neoplasms," Cancer Causes \& Control, vol. 28, no. 2, pp. 107-115, 2017.

[3] B. Croft, M. Reed, C. Patrick, N. Kovacevich, and I. A. Voutsadakis, "Diabetes, obesity, and the metabolic syndrome as prognostic factors in stages I to III colorectal cancer patients," Journal of Gastrointestinal Cancer, vol. 50, no. 2, pp. 221-229, 2019.

[4] T. Matsuda, H. M. Chiu, Y. Sano, T. Fujii, A. Ono, and Y. Saito, "Surveillance colonoscopy after endoscopic treatment for colorectal neoplasia: from the standpoint of the Asia-Pacific region," Digestive Endoscopy, vol. 28, no. 3, pp. 342-347, 2016.

[5] H. Jayasekara, D. R. English, A. Haydon et al., "Associations of alcohol intake, smoking, physical activity and obesity with survival following colorectal cancer diagnosis by stage, anatomic site and tumor molecular subtype," International Journal of Cancer, vol. 142, no. 2, pp. 238-250, 2018.

[6] L. Zhang, S. B. Kim, K. Luitel, and J. W. Shay, "Cholesterol depletion by TASIN-1 induces apoptotic cell death through the ER stress/ROS/JNK signaling in colon cancer cells," Molecular Cancer Therapeutics, vol. 17, no. 5, pp. 943-951, 2018, Epub 2018/02/23.

[7] F. A. Sinicrope, N. R. Foster, D. J. Sargent, M. J. O'Connell, and C. Rankin, "Obesity is an independent prognostic variable in colon cancer survivors," Clinical Cancer Research, vol. 16, no. 6, pp. 1884-1893, 2010.

[8] C. A. Doubeni, D. A. Corley, V. P. Quinn et al., "Effectiveness of screening colonoscopy in reducing the risk of death from right and left colon cancer: a large community-based study," Gut, vol. 67, no. 2, pp. 291-298, 2018.

[9] J. Tong, Y. Wang, B. Chang, D. Zhang, and B. Wang, "Associations between tumor markers and the risk of colorectal polyp recurrence in Chinese people," International Journal of Clinical and Experimental Medicine, vol. 8, no. 4, pp. 6397-6405, 2015.

[10] K.-C. Hu, M.-S. Wu, C.-H. Chu et al., "Decreased colorectal adenoma risk after Helicobacter pylori eradication: a retrospective cohort study," Clinical Infectious Diseases, vol. 68, no. 12, pp. 2105-2113, 2018.

[11] J. L. Wang, X. Liang, J. Xu, Y. X. Chen, and J. Y. Fang, "Helicobacter pylori infection increases the risk of colorectal adenomas: an updated meta-analysis," Clinical Laboratory, vol. 64, no. 7, pp. 1163-1170, 2018.

[12] Y. M. ChangxiChen, J. Du, Y. Xu, Z. Zhu, and H. Cao, "Helicobacter pylori infection associated with an increased risk of colorectal adenomatous polyps in the Chinese population," BMC Gastroenterology, vol. 19, no. 1, p. 14, 2019.

[13] N. Zumkeller, H. Brenner, M. Zwahlen, and D. Rothenbacher, "Helicobacter pylori infection and colorectal cancer risk: a meta-analysis," Helicobacter, vol. 11, no. 2, pp. 75-80, 2006.

[14] S. Y. Oh, D. I. Park, T. W. Yoo et al., "Is gastric cancer a new indication for surveillance colonoscopy? Colon cancer is increased in gastric cancer patients," The Korean Journal of Gastroenterology, vol. 47, no. 3, pp. 191-197, 2006.

[15] R. K. Siddheshwar, K. B. Muhammad, J. C. Gray, and S. B. Kelly, "Seroprevalence of Helicobacter pylori in patients with colorectal polyps and colorectal carcinoma," The American Journal of Gastroenterology, vol. 96, no. 1, pp. 84-88, 2001. 
[16] K. Abbass, W. Gul, G. Beck, R. Markert, and S. Akram, "Association of Helicobacter pylori infection with the development of colorectal polyps and colorectal carcinoma," Southern Medical Journal, vol. 104, no. 7, pp. 473-476, 2011.

[17] J. Butt, M. G. Varga, W. J. Blot et al., "Serologic response to Helicobacter pylori proteins associated with risk of colorectal cancer among diverse populations in the United States," Gastroenterology, vol. 156, no. 1, pp. 175-186.e2, 2019.

[18] Y. L. Lin, J. K. Chiang, S. M. Lin, and C. E. Tseng, "Helicobacter pylori infection concomitant with metabolic syndrome further increase risk of colorectal adenomas," World Journal of Gastroenterology, vol. 16, no. 30, pp. 3841-3846, 2010, Epub 2010/08/11.

[19] J. Raisch, N. Rolhion, A. Dubois, A. Darfeuille-Michaud, and M. A. Bringer, "Intracellular colon cancer-associated Escherichia coli promote protumoral activities of human macrophages by inducing sustained COX-2 expression," Laboratory Investigation, vol. 95, no. 3, pp. 296-307, 2015, Epub 2014/12/30.

[20] K. Echizen, O. Hirose, Y. Maeda, and M. Oshima, "Inflammation in gastric cancer: interplay of the COX-2/prostaglandin E2 and Toll-like receptor/MyD88 pathways," Cancer Science, vol. 107, no. 4, pp. 391-397, 2016.

[21] L. B. Sansbury, R. C. Millikan, J. C. Schroeder, P. G. Moorman, K. E. North, and R. S. Sandler, "Use of nonsteroidal antiinflammatory drugs and risk of colon cancer in a populationbased, case-control study of African Americans and Whites," American Journal of Epidemiology, vol. 162, no. 6, pp. 548558, 2005.

[22] M. Selgrad, J. Bornschein, A. Kandulski et al., "Helicobacter pylori but not gastrin is associated with the development of colonic neoplasms," International Journal of Cancer, vol. 135, no. 5, pp. 1127-1131, 2014.

[23] J. P. Im, D. Kim, S. J. Chung et al., "Visceral obesity as a risk factor for colorectal adenoma occurrence in surveillance colonoscopy," Gastrointestinal Endoscopy, vol. 88, no. 1, pp. 119-127.e4, 2018.

[24] N. Fliss-Isakov, S. Zelber-Sagi, M. Webb, Z. Halpern, O. Shibolet, and R. Kariv, "Distinct metabolic profiles are associated with colorectal adenomas and serrated polyps," Obesity, vol. 25, pp. S72-S80, 2017.

[25] A. Kushiyama, Y. Nakatsu, Y. Matsunaga et al., "Role of Uric Acid Metabolism-Related Inflammation in the Pathogenesis of Metabolic Syndrome Components Such as Atherosclerosis and Nonalcoholic Steatohepatitis," Mediators of Inflammation, vol. 2016, 15 pages, 2016.

[26] F. Dovell and P. Boffetta, "Serum uric acid and cancer mortality and incidence," European Journal of Cancer Prevention, vol. 27 , no. 4, pp. 399-405, 2018 Jul;. 


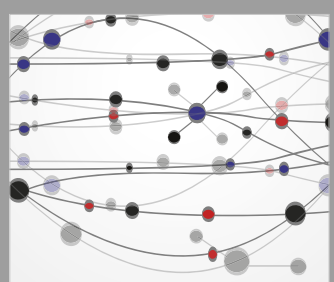

The Scientific World Journal
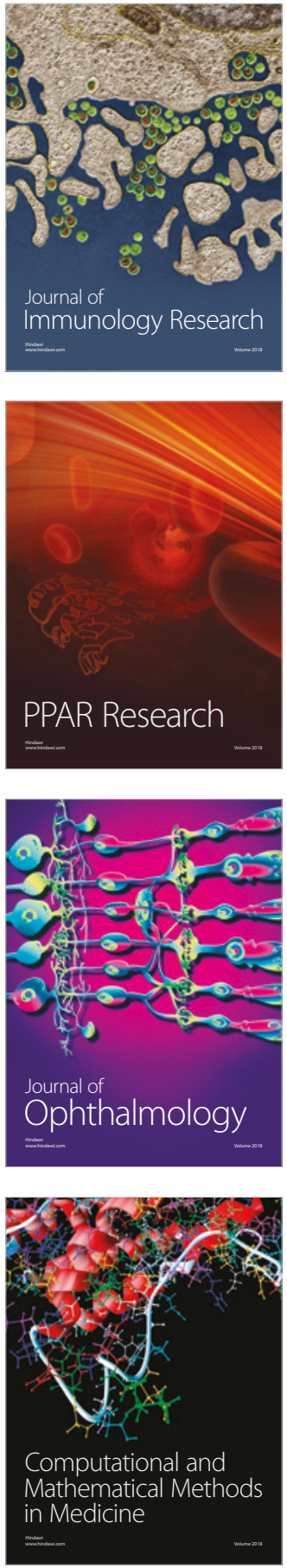

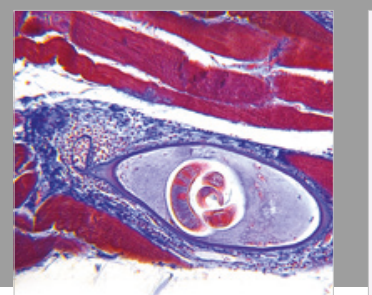

Gastroenterology Research and Practice

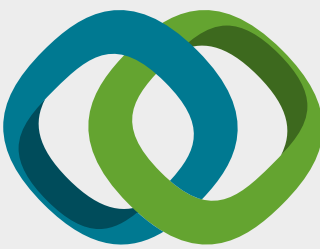

\section{Hindawi}

Submit your manuscripts at

www.hindawi.com
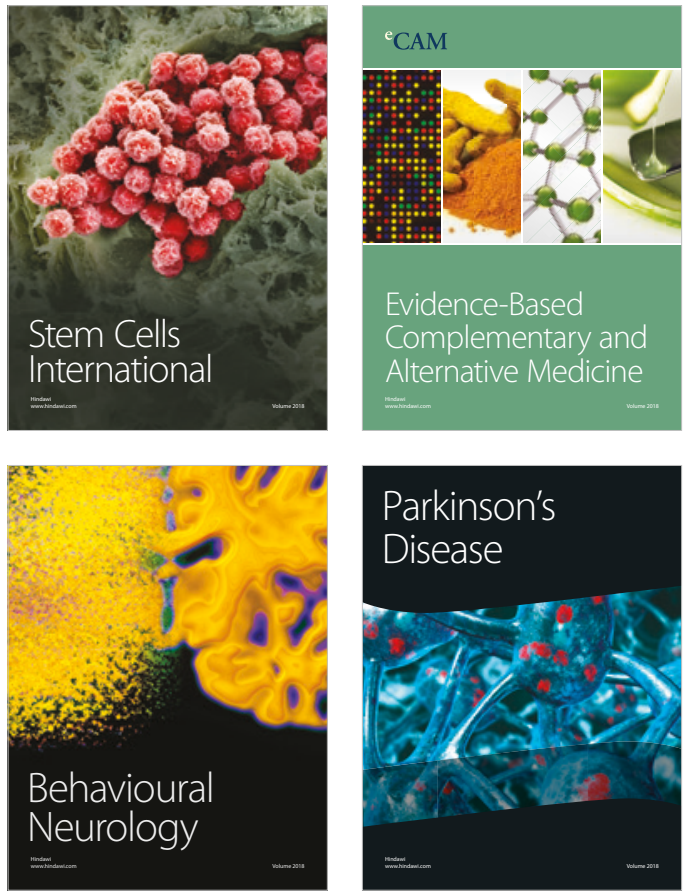

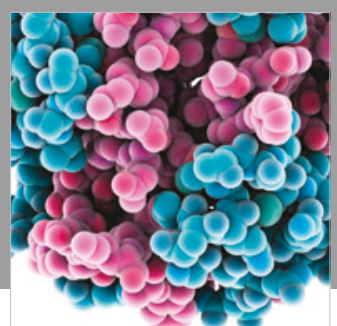

ournal of

Diabetes Research

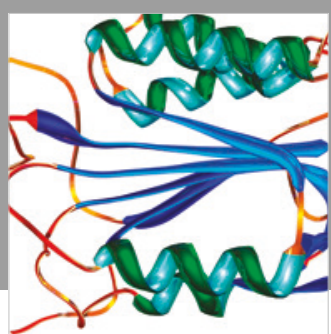

Disease Markers
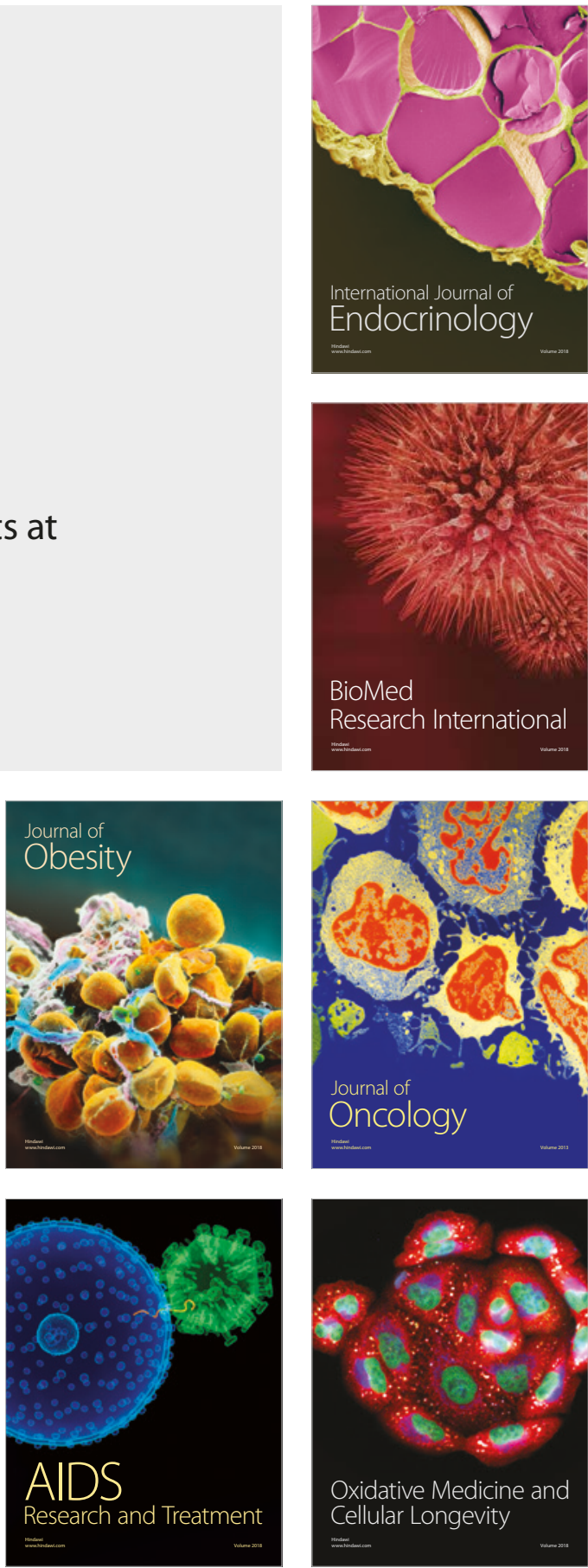